\title{
Tests of two explanations of response elimination by noncontingent reinforcement
}

\author{
H. M. JENKINS and W. A. LAMBOS \\ McMaster University, Hamilton, Ontario, Canada
}

\begin{abstract}
Two experiments are reported on the elimination of autoshaped keypecking in pigeons by introducing added feedings that have the effect of removing the contingency between keylight (CS) and feedings. In Experiment 1, added feedings were signaled, by an already conditioned stimulus, in one group but not in another. Contrary to the Rescorla-Wagner theory, but consistent with scalar expectancy theory, responding in these groups declined at equal rates. In Experiment 2, following acquisition, some groups were exposed to repeated sessions in which they received feedings alone prior to receiving both CS and feedings noncontingently. Although prior exposure to feedings did reduce the total amount of responding in subsequent noncontingent sessions, it did not, contrary to scalar expectancy theory, reduce the initial level of responding to the CS. It is suggested that a differential between reinforcing conditions in the CS as compared with neighboring non-CS periods is more fundamental to conditioning than is acknowledged in the Rescorla-Wagner theory or in scalar expectancy theory.
\end{abstract}

Responding ceases when the stimulus-reinforcer contingency is removed following the acquisition of a classically conditioned response. Gamzu and Williams $(1971,1973)$ and Lindblom and Jenkins $(1981)$ have shown this to be true for the classically conditioned response of autoshaped keypecking in the pigeon. In these experiments, the stimulus-reinforcer contingency was removed by the addition of a sufficient number of reinforcers in the absence of the stimulus to bring the average wait between reinforcers (equivalently, the average rate or probability of the reinforcer) to a value that was the same in either the presence or the absence of the keylight (CS). Reinforcers in the presence of the CS continued as in acquisition so that the contiguity between CS and reinforcer was not altered. The cessation of responding under these conditions shows that more than CS-US contiguity is involved in the maintenance of classically conditioned responding. Two major theories have attempted to specify what, beyond contiguity, might be involved.

The Rescorla-Wagner theory (1972) provides one account of the process responsible for the cessation of responding. The essential points, which follow from the basic equations of the theory, are that (1) the autoshaping stimulus, like any other CS, is presented in the context of ever-present background stimuli; (2) the introduction of intertrial reinforcers makes

This research was supported by a grant to the first author from the National Science and Engineering Research Council of Canada. Portions of this report were presented at the meeting of the Psychonomic Society, Minneapolis, 1982. Requests for reprints should be sent to the first author, Department of Psychology, McMaster University, Hamilton, Ontario L8S 4K1, Canada. the background excitatory; (3) as a result, the total signal value of the compound consisting of the background and the autoshaping stimulus comes to exceed the maximum value supportable by the reinforcer; (4) the autoshaping stimulus and the background then lose strength on each trial even though the reinforcer continues to occur in conjunction with the autoshaping stimulus; and (5) the background regains signal value during the intertrial periods as a result of the intertrial reinforcers and therefore continues to drive the signal value of the autoshaping stimulus toward zero on each trial-in the limit, the background obtains all the signal value, while the autoshaping stimulus is completely neutralized.

It is important to recognize that, according to the Rescorla-Wagner theory, conditioning occurs as a consequence of the contiguity between a stimulus and a reinforcer provided only that there is not a concurrent stimulus that is fully conditioned as a signal of the reinforcer. Contingency between stimulus and reinforcer is not taken as a fundamental condition for the acquisition of signal value. Rather, the effect of contingency is accounted for through the concept of competition between background and CS for a limited pool of strength. The theory can be characterized as a contiguity-plus-competition theory.

An alternative account for the cessation of responding when the stimulus-reinforcer contingency is removed is provided by the application of scalar expectancy theory to classical conditioning (Gibbon \& Balsam, 1981; Jenkins, Barnes, \& Barrera, 1981). According to that theory, performance is governed by a ratio comparison of the average wait for food in the presence of the CS with the average wait across the entire experimental setting without regard to the 
stimulus conditions that accompany feeding, specifically, without regard to whether feedings are or are not signaled by a CS. According to scalar expectancy theory, the introduction of reinforcers during the intertrial period at the same average rate of presentation as in the presence of the CS results in the cessation of responding, because the ratio of the average wait per reinforcer during the stimulus to the average wait overall is made equal to one.

Like the Rescorla-Wagner theory, scalar expectancy theory is fundamentally a contiguity theory of conditioning, although not of a traditional variety. Reinforcer expectation in the stimulus develops, according to the theory, as a consequence of the contiguity, or occurrence together in time, of the stimulus with an average rate of occurrence of the reinforcer. Performance, but not association, depends on a comparison between the average wait per reinforcer in the stimulus with the overall average wait in the experimental setting. The theory might be characterized as a contiguity-plus-comparison theory, as distinct from a contiguity-plus-competition theory of the Rescorla-Wagner type.

According to the Rescorla-Wagner theory, a reinforcer that is signaled by an asymptotically conditioned stimulus no longer contributes excitatory strength to the background; a fully conditioned stimulus blocks conditioning to background stimuli. Consequently, if intertrial reinforcers were signaled by a fully conditioned stimulus, responding to the trial stimulus should continue, even though the stimulus-reinforcer contingency was removed by the added intertrial reinforcers. In contrast, if the added reinforcers are unsignaled, responding should cease. According to scalar expectancy theory, however, signaling the intertrial reinforcers should have no effect. Responding should cease at the same rate whether the added reinforcers are signaled or not, because the waiting time in the stimulus would equal the overall waiting time. These implications were tested in Experiment 1.

\section{EXPERIMENT 1}

\section{Method}

Subjects. Thirty-six, experimentally naive, unsexed, adult homing pigeons, maintained at $80 \%$ of free-feeding weight, served as subjects. The colony room was lighted from 7 a.m. to 9:30 p.m. Subjects were run between the hours of 1 and 5 p.m., one session per day, 6 days per week.

Apparatus. Six modified Lehigh Valley pigeon chambers were used. They were equipped with a speaker, a solenoid-operated feeder tray, and a ventilating fan. The front panel contained two response keys; only the left key was used. The key, which operated on a force of $15 \mathrm{~g}(.145 \mathrm{~N})$, was a square of hinged plastic, $2.5 \mathrm{~cm}$ on a side. At its center was a circle, $7 \mathrm{~mm}$ in diameter, which could be backlighted by red or green light. The center of the key was $20 \mathrm{~cm}$ above the chamber floor and $16 \mathrm{~cm}$ from the left edge of the front panel. Operation of the feeder tray made available mixed grain through an opening, $5.7 \times 5 \mathrm{~cm}$, centered on the front panel. The lower edge of the opening was $4.8 \mathrm{~cm}$ above the floor. A white lamp (GE 1829) illuminated the opening during the tray operation. The chamber was continuously illuminated by a row of three lamps (GE 1829) mounted across the top of the front panel in housings that directed the light to the ceiling. Masking noise was continuously present. Programming and recording were accomplished by a Commodore PET computer located in an adjacent room.

Procedure. The design of Experiment 1 is shown in Figure 1. During acquisition, separate sessions of autoshaping to a red keylight alternated daily with autoshaping to a green keylight. The programming of sessions was on the basis of 12-sec periods. Each of the first 26 sessions of acquisition consisted of 2012 -sec keylighton periods and 20012 -sec keylight-off (intertrial) periods in a random sequence. There was a 4-sec feeding during the last $4 \mathrm{sec}$ of each 12 -sec keylight-on period. The final 34 sessions of acquisition consisted of 50 keylight-on periods and 50 keylight-off periods in a random sequence. A randomly selected $40 \%$ of the keylighton periods contained a 4 -sec feeding during the last $4 \mathrm{sec}$ of the 12 -sec period. In effect, the keylight-on periods were of variable duration, with a timing modulus of $12 \mathrm{sec}$, and reinforcement occurred with a probability of .4 in each $12-\mathrm{sec}$ stimulus.

At the end of acquisition, nine unreliable responders were eliminated as subjects. It may be noted that, on the basis of scalar expectancy theory, the parameters used in the latter part of the acquisition phase would not be expected to maintain a very high level of autoshaped responding. The remainder were divided into three groups of nine subjects each, matched on the basis of keypecking rates in the last five sessions of acquisition. Subjects in Group $R \bullet G \bullet$ received sessions in which all intertrial periods with the unlit key were removed, and the red and green keylight stimuli, with their associated feedings, were interleaved and filled the entire session. As a result, half of the $12-\mathrm{sec}$ periods were red and half were green, in random sequence. Forty percent of the 12-sec periods contained a $4-\mathrm{sec}$ feeding during the last $4 \mathrm{sec}$. Subjects in Group $(-) \bullet G$. also received sessions in which all the feedings formerly given in separate sessions now occurred in a single session, but only one of the keylight stimuli was retained. This is the typical noncontingent arrangement. For subjects in Group

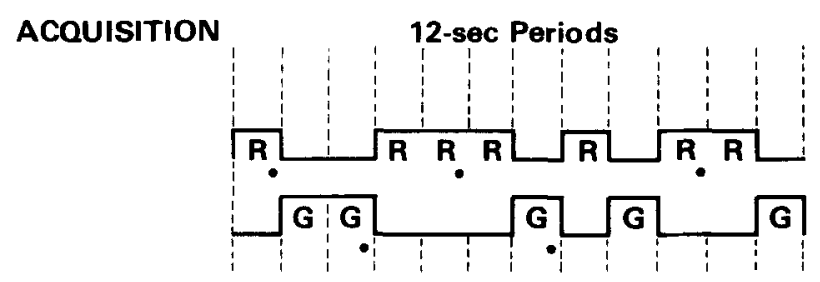

TREATMENTS Group RG.
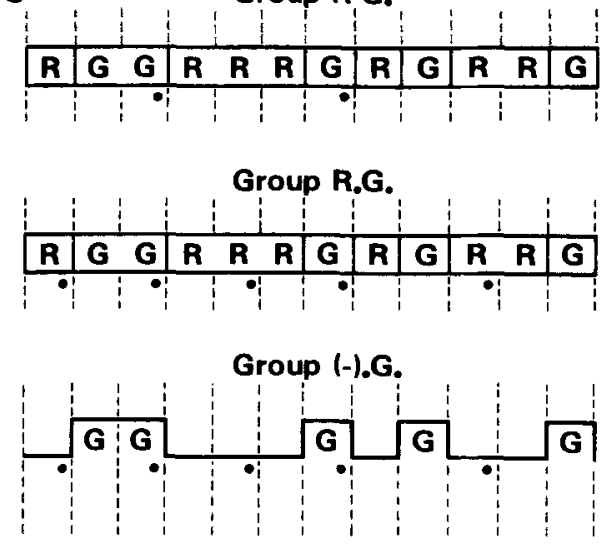

Figure 1. Design of Experiment 1. The location of feedings is shown by dots. $R$ stands for red lighted key, $G$ for green. 
RG•, the stimuli were retained when the sessions were combined, but all feedings were dropped from one stimulus. Although the group designations are written as if only the green keylight was used as the reference trial stimulus, the experiment was counterbalanced for stimulus colors in the treatment phase. In Group R•G•, data for the rate of responding during the treatment phase are based on individual mean values for the red and green keylight stimuli.

According to the Rescorla-Wagner theory, responding should be maintained in Group $R \bullet G \bullet$ but not in Group (-)•G•. According to scalar expectancy theory, responding should cease, and at the same rate, in these groups. Both theories predict the maintenance of responding in Group $\mathbf{R}$ G. This group differs from Group $R \bullet G \bullet$ only in that feedings in the red keylight stimulus are absent, which allows an assessment of the effect of the extra feedings under identical stimulus conditions.

\section{Results and Discussion}

The median percent of trials responded to for the 27 subjects that went through all phases is shown for the acquisition and treatment phases in Figure 2. In acquisition, there was a slight reduction in the percent of trials responded to after the change made in the programming of trials beginning with Session 27. The change consisted of the addition of 30 nonreinforced 12-sec keylight-on periods and the removal of 150 12-sec keylight-off, or intertrial, periods. From this point on, the keylight-on periods constituted half of each session. Nevertheless, at the end of the acquisition phase the median percentage of trials responded to was in excess of 90 .

Throughout the treatment phase in Group R G., responding to the reinforced keylight stimulus was maintained, whereas responding to the unreinforced keylight declined to a low level. In Groups R.G• and $(-) \bullet G_{\bullet}$, the percentage of trials responded to declined almost to zero. There was no difference in the rate or extent of decline in these groups.

Comparable data on rate of responding are shown in Figure 3. These data also show no difference in the rate of decline of responding during the treatment phase for Group (-).G• as compared with Group R.G.. Rate of responding does show, however, one effect that could not be seen in the measure of percentage of trials responded to-namely, a substantial, and highly significant, elevation of responding to the reinforced keylight in Group R G. based on a comparison of mean rates for the last 10 sessions of acquisition and the first 10 sessions of the treatment phase $[t(8)=4.68, p<.002]$. This is an interesting example of a behavioral-contrast-like effect, because it occurs with no change from the baseline condition in the overall rate or distribution of feedings. The contrast-like-effect was obtained by the replacement of the intertrial, keylight-off periods with one of the previously reinforced, but now unreinforced, keylight stimuli. It cannot be said, however, that the effect depends on the previous reinforcement of the stimulus, because it might be due merely to the change from an unlit key to a lighted key between trials. The elevation of responding observed in Group $R$.

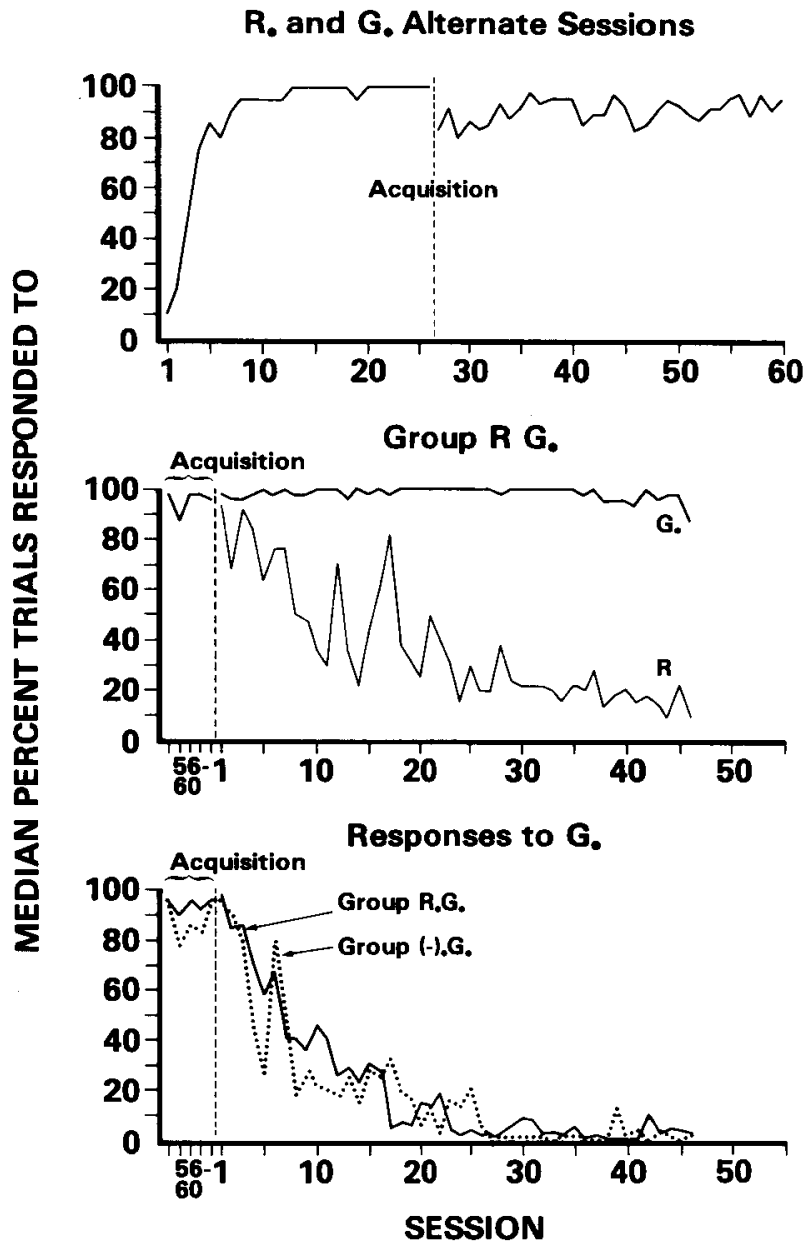

Figure 2. Percentage of trials responded to during acquisition and treatment sessions, Experiment 1 . The dotted line in the upper panel marks a change in the acquisition parameters (see text). Although the labeling of groups suggests that the green keylight always served as the reference $\mathrm{CS}$, the experiment was counterbalanced for key colors.

predicted by neither the Rescorla-Wagner theory nor the scalar expectancy theory.

Contrary to the Rescorla-Wagner theory, removal of the stimulus-reinforcer contingency under conditions that should prevent conditioning to background stimuli resulted in a loss of responding. Even if the background is assumed to be far more salient than the keylight stimulus, the theory predicts no loss in the excitatory values of the stimuli in the treatment phase when all reinforcers continue to be signaled by the previously conditioned keylight stimuli (Group $\left.R_{\bullet} G_{\bullet}\right)$. Although some modification of the basic equations in the theory might yield a prediction of loss of responding for this case, it is difficult to conceive of a way to preserve the fundamental assumption of competition between conditioning to background and conditioning to $\mathrm{CS}$ in view of the ob- 


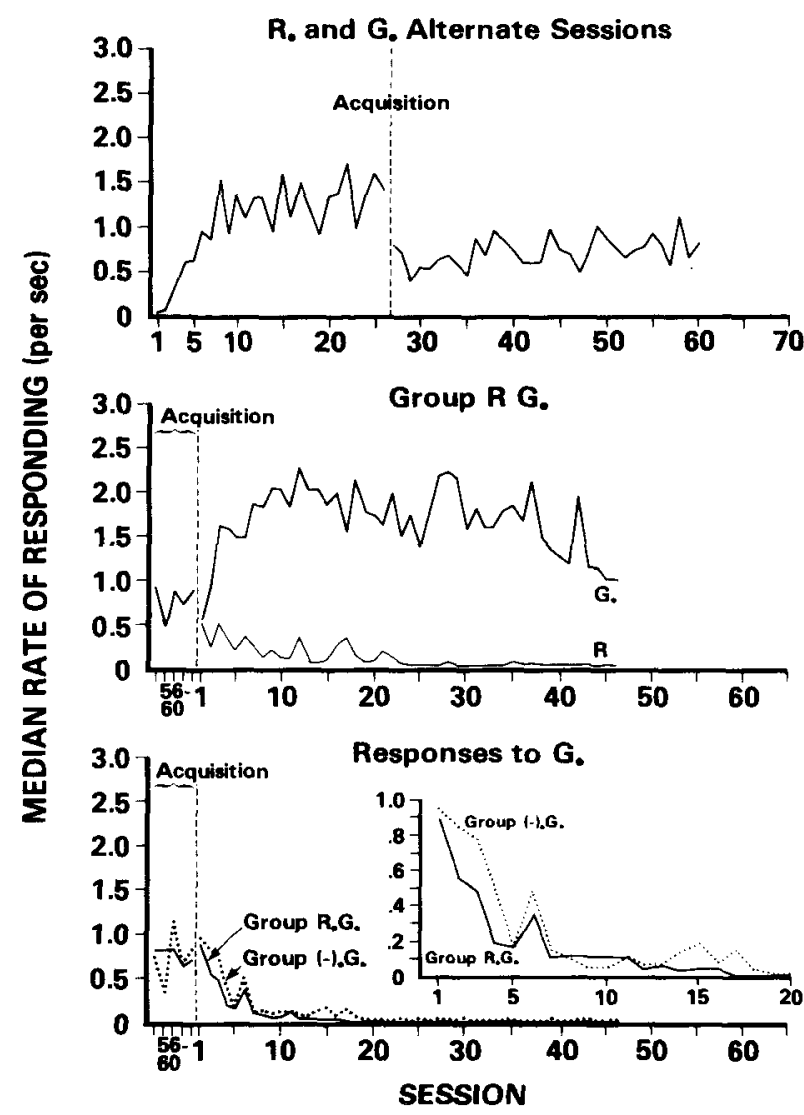

Figure 3. Rate of responding on trials during acquisition and treatment sessions. Insert at lower right replots, on an expanded scale, data in lower panel for first 20 treatment sessions.

served lack of a difference in the rate of decline in responding when the added reinforcers are, or are not, signaled by previously conditioned stimuli. The results of another experiment (Jenkins et al., 1981, Experiment 13) suggest that acquisition of autoshaped responding is also affected equally by the addition of signaled and unsignaled feedings.

The observed equality in the rate of decline of responding when the stimulus-reinforcer contingency was removed by the addition of signaled or unsignaled reinforcers is consistent with scalar expectancy theory. In either case, the average waiting time in the stimulus is made equal to the overall waiting time in the session, and therefore no difference in the rate of decline in responding is predicted.

\section{EXPERIMENT 2}

We turn now to another case involving the removal of the stimulus-reinforcer contingency, for which the Rescorla-Wagner model and the scalar expectancy theory make clearly divergent predictions. According to scalar expectancy theory, the excitatory value of the CS should be removed by the following procedure: With the previously conditioned CS withdrawn, reinforcers are presented at an interreinforcement interval equal to that which obtained in the CS. Given sufficient exposure to such a regime, the expected waiting time for the reinforcer in the absence of CS, and overall, should come to equal the previously established expectation in the presence of the CS. Therefore, upon reintroduction of the CS, responding should be at the same low level as would be expected from an exposure to the usual noncontingent procedure in which the CS continues to be presented together with equal reinforcement in the intertrial periods.

The Rescorla-Wagner theory, on the other hand, predicts that, although the delivery of reinforcers with the CS removed should hasten the loss of responding once the CS is reintroduced, the initial level of response on the first reintroduction of CS should not be reduced when compared with the level at the end of acquisition. Only with repeated presentations of the CS in the presence of noncontingency does the CS lose excitatory value according to the Rescorla-Wagner theory. These differential predictions were tested in Experiment 2.

\section{Method}

Subjects and Apparatus. Eighteen homing pigeons of the type used in Experiment 1 were used in the same apparatus.

Procedure. All subjects received acquisition training consisting of 21 sessions of autoshaping to a red keylight. As in Experiment 1, a 12-sec programming modulus was used. In each of the first 11 sessions there were 25 keylight-on and 195 keylight-off, or intertrial periods. Ten of the keylight-on periods, or $40 \%$, contained a 4-sec feeding in the last $4 \mathrm{sec}$. During the last 10 sessions of acquisition, the number of keylight-off periods was reduced to 75, while the number of keylight-on periods remained at 25 .

Following acquisition, three subjects were discarded as unreliable responders. The remainder were divided into three matched groups of five subjects each, based on response rates averaged over the last five sessions of acquisition. Beginning with Session 22 , each group received 27 sessions in which a random $40 \%$ of 100 12-sec periods were reinforced with a 4-sec feeding. Thus, the probability of reinforcement throughout the entire session was the same as the probability of reinforcement for the keylight-on periods of acquisition. For subjects in Group $F_{21} \rightarrow F / S$, the first 21 sessions contained only keylight-off periods. During the last 6 sessions, a random $40 \%$ of all periods continued to be reinforced, but there were now 25 keylight-on periods (CS) and 75 keylight-off periods (noncontingent reinforcement of the CS). For the subjects in Group $F_{1 s} \rightarrow F / S$, the first 15 sessions contained only keylight-off periods. During the last 12 sessions, the CS was reinforced noncontingently under the same conditions as described for the previous group. Group F/S received no prior sessions with keylight-off periods. Subjects in this group went directly to sessions with the CS noncontingently reinforced under the conditions described above. They received 27 such sessions.

Following the last session of noncontingent reinforcement of the CS, subjects received 20 further sessions in which the only change was the complete removal of food. The purpose was to test for a recovery of responding of the kind previously obtained under similar conditions by Lindblom and Jenkins (1981). The first recovery-test session began with the feeding schedule of the previous noncontingent reinforcement sessions in place until, on 
average, eight keylight-on periods had been received. Thereafter, the food tray was disconnected. No feedings were received at any point in the remaining recovery-test sessions.

\section{Results and Discussion}

Response rates during acquisition and in the subsequent noncontingent sessions are shown in Figure 4. Following the 11 th acquisition session, the number of intertrial periods was reduced, but this had no apparent effect on response rate to the CS. The results of principal interest concern performance in the noncontingent sessions.

As is apparent in Figure 4, noncontingent reinforcement of the CS resulted in a rapid reduction of the median response rate to zero in each group. The effect of prior exposure to feedings in the absence of the CS was clearest in the first session of noncontin-

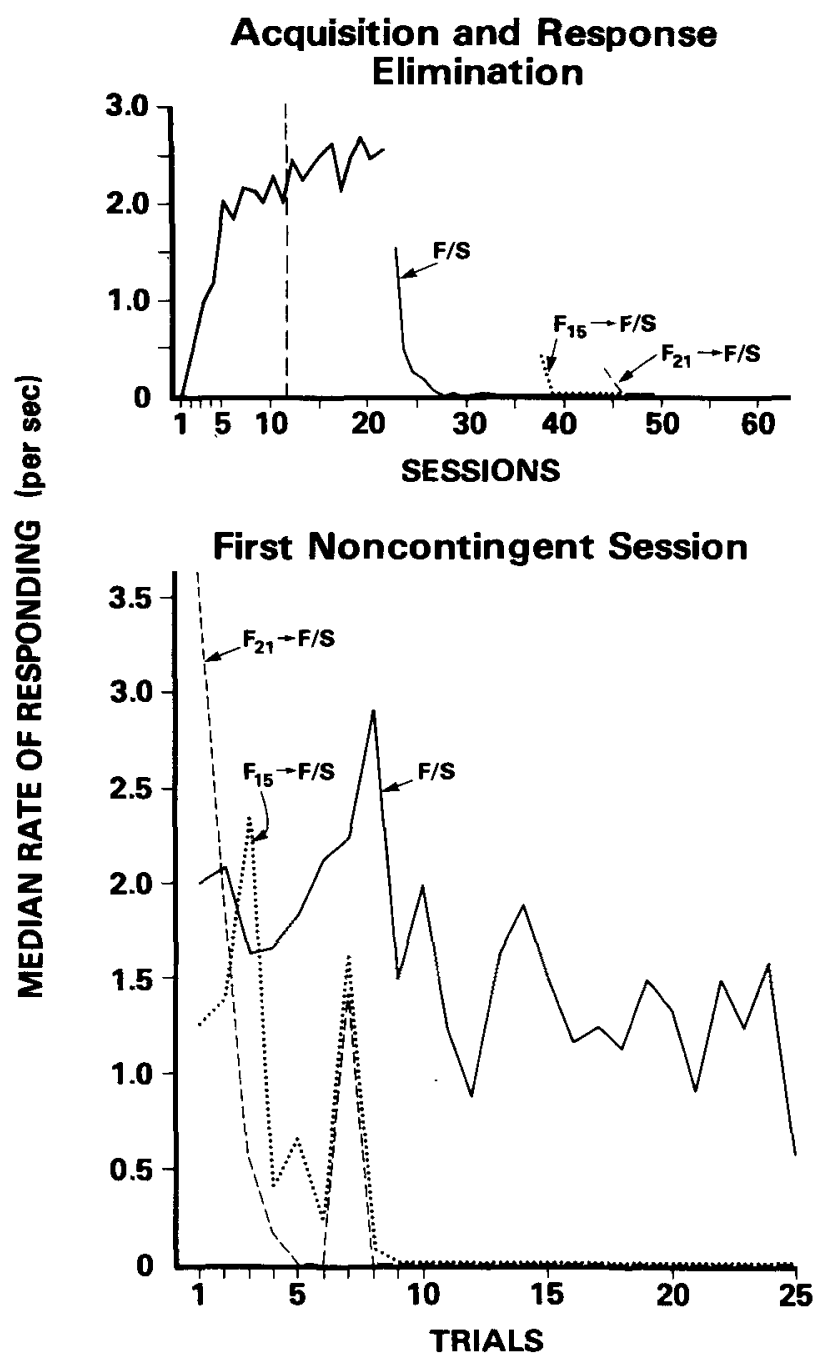

Figure 4. Upper panel shows rate of responding on trials during acquisition and response-elimination sessions, Experiment 2. Lower panel shows response rate by individual trials during the first session of noncontingent CS presentations. gent reinforcement of the CS. The trial-by-trial record of responding for that session is shown in the lower panel of Figure 4. The very first CS presentation was responded to in each group at approximately the same rate as the CS was at the end of the acquisition phase. Analysis of variance of the mean response rates on the first presentation showed no hint of an effect $(F<1)$ due to the extensive prior exposure to feedings received by Groups $F_{15} \rightarrow F / S$ and $F_{21} \rightarrow F / S$ in the absence of the CS. The decline in response rate over successive CS presentations was, however, more rapid in each of these groups than in Group F/S, which received no prior sessions of feedings in the absence of the CS. The less rapid rate of decline in Group $\mathrm{F} / \mathrm{S}$ was reflected in a significant interaction of groups and trials $[\mathrm{F}(48,288)=1.58, \mathrm{p}<.025]$. Since the decline in response rate over trials in Groups $F_{15} \rightarrow F / S$ and $F_{21} \rightarrow F / S$ was statistically very similar, the interaction reflects the difference between Group F/S and the other two groups. By the end of the first session, the median response rates for subjects in Groups $F_{13} \rightarrow F / S$ and $F_{21} \rightarrow F / S$ had dropped to zero. In Group $F / S$, however, the median response rate was still substantial. The median test (Hays, 1981), based on response rates averaged over the last five trials, was highly significant $(\mathrm{p}<.005)$.

Although the decline in responding to a CS brought about by noncontingent reinforcement of the CS is a replicable finding, it is worth noting that some subjects persist in responding to CS (and not in its absence) for many sessions. In the present experiment, one subject in each group showed such persistence throughout all sessions of noncontingency. The effect of noncontingent reinforcement on previously acquired autoshaped responding is highly variable.

The effect of the removal of food on responding to the CS within the first recovery-test session is shown in Figure 5. All groups showed a similar strong recovery in responding. In order to assess the extent of recovery, we selected, for individual subjects, the block of five trials with the highest rates during the last session of acquisition and did the same for the first two sessions of recovery. The groups did not differ significantly on these measures. The overall mean rate for the acquisition block was 2.47 responses/ $\sec (\mathrm{SD}=1.12)$. The comparable figure for recovery sessions was 2.84 responses $/ \mathrm{sec}(\mathrm{SD}=1.44)$. Recovery appears to have been complete. Responding did not return to the level it had reached at the end of the noncontingent sessions until about eight extinction sessions had been given. The course of responding over extinction sessions was highly similar among the groups, and there were no significant differences in total number of responses in the 20 extinction sessions $(F<1)$.

The results of this experiment are not in agreement with the prediction of the scalar expectancy theory. The extensive exposure in Groups $F_{15} \rightarrow F / S$ and 


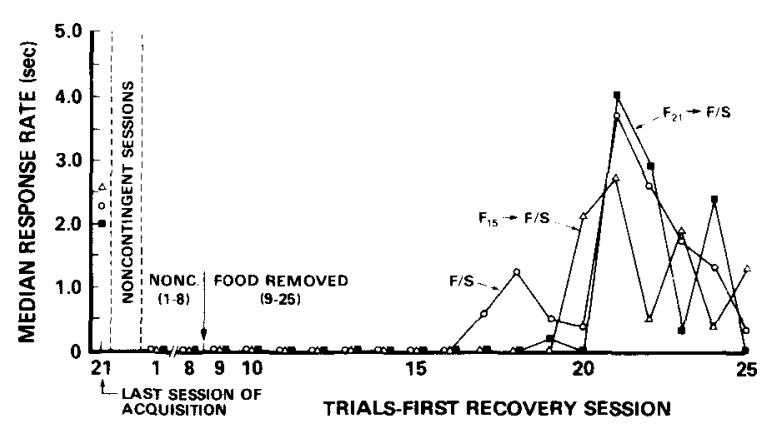

Figure 5. Response rate by individual trials of the first recovery session.

$F_{21} \rightarrow F / S$ to sessions with feedings only would be expected, on the basis of that theory, to greatly reduce the initial level of responding to the CS in the noncontingent sessions because the expected waiting time per feeding in the absence of the CS, and overall, should have been raised to much the same value as the previously established waiting time in the presence of the CS. The finding of no effect on the initial level of responding indicates that something other than a comparison of expected waiting times is involved when the noncontingent procedure results in the cessation of responding.

The Rescorla-Wagner theory predicts, in agreement with present results, that equating the feeding rate in the absence of the CS to the feeding rate in the presence of the CS will not reduce the initial level of response to the CS but will result in a more rapid decline in responding. According to the theory, the CS must be presented concurrently with the reinforced background in order for its excitatory value to be reduced. The reduction will take place more rapidly because, in theory, the background will be conditioned at the outset and will therefore begin to remove signal value from the $C S$ following the very first CS presentation.

There is some ambiguity in the application of the Rescorla-Wagner theory to autoshaped responding; specifically, it is not clear whether the strength of the autoshaped response should be taken to be a function of the total signal strength of background plus CS or only of the CS. With the former interpretation one might expect, depending on what is assumed about the rate of background conditioning, that the initial level of response to the CS would be higher in those subjects that received sessions of USalone presentations than in those that did not. Considering the interpretive latitude allowed by the theory, the absence of a significant difference among the groups in the initial response to the CS in the present experiment does not pose a problem for the theory. On the other hand, the strong recovery of responding that resulted from the complete removal of food does pose a problem. According to the theory, exposure to noncontingency should remove signal value from the CS and reconditioning should therefore be necessary for recovery (see Lindblom \& Jenkins, 1981).

\section{CONCLUDING DISCUSSION}

Predictions from two theories that offer explanations for the cessation of responding that occurs when the stimulus-reinforcer contingency is removed were tested. The Rescorla-Wagner theory predicts that when the added reinforcers that remove a stimulusreinforcer contingency are signaled, the background should be protected from conditioning and, therefore, responding to CS should be maintained. In Experiment 1 , we found, however, that responding was eliminated under these conditions. Moreover, it was eliminated at the same rate as it was when the added reinforcers were unsignaled. These results were in accord with scalar expectancy theory.

Experiment 2 examined the effect that repeated feedings in the absence of the CS had on subsequent responding to the $\mathrm{CS}$. According to scalar expectancy theory, repeated feedings in the absence of CS should greatly reduce, if not eliminate, responding to CS. The results of Experiment 2, however, showed no reduction in the initial level of responding to the CS due to such feedings.

The effect of US-alone presentations on subsequent responding to a previously conditioned CS has been examined in taste-aversion conditioning (Brookshire \& Brackbill, 1976; Holman, 1976; Riley, Jacobs, \& LoLordo, 1976) and in fear conditioning (Ayres \& Benedict, 1973). The results of these experiments are consistent in showing that US-alone presentations do not reduce subsequent responding to the CS. Although the present results showed no effect of US-alone presentations on the initial level of responding to $C S$, they did show an effect of USalone presentations on the subsequent rate of decline in responding. It is important to note that the test procedure in the present experiment differs from that of previous experiments. We tested in the context of noncontingent reinforcement of the CS, whereas in previous experiments the test was made with the US removed. When the test is made with the US removed, recovery from the effects of prior US-alone presentations might occur. That possibility is suggested by the fact that autoshaped responding recovers from the suppressive effects of noncontingent reinforcement of the CS when the US is removed. Accordingly, the difference in testing procedures might be responsible for the discrepancy between the present finding of a more rapid decline in responding due to US-alone presentations and previous findings of no reduction in responding due to US-alone presentations.

The present experiments point to difficulties in currently available explanations for the elimination of the responding that results from noncontingent reinforcement of a previously conditioned CS. These 
difficulties might have a common source. As we have noted, both the Rescorla-Wagner theory and the scalar expectancy theory seek, in different ways, to account for the demonstrated importance of CS-US contingency while preserving the time-honored contiguity assumption. Perhaps adherence to the contiguity assumption underlies the difficulties of each of these theories in dealing with the present observations.

The contiguity assumption assigns no role in establishing a CS-US association to a differential in any parameter of reinforcement in the presence of the CS as compared with its absence. On the contiguity assumption, association is accounted for in terms of the occurrence of reinforcers within the boundaries of the CS presentation. It would violate the contiguity assumption to speak of contiguity in terms of a change in the stimulus and a change in the occurrence of USs, because to do so would entail a differential in some parameter of reinforcement within the CS as opposed to before or after the CS. That would run counter to the point of the contiguity assumption in the Rescorla-Wagner and the scalar expectancy theories, which is intended to account for association without reference to the differential reinforcement of CS and non-CS periods.

The results of Experiment 1 suggest that autoshaped responding cannot be maintained unless there is a reinforcement differential between CS and nonCS. When this differential was removed, responding was eliminated with equal rapidity regardless of whether the stimulus conditions would, according to the Rescorla-Wagner theory, prevent or allow competition between background and CS. Furthermore, the results of Experiment 2 suggest that autoshaped responding cannot be eliminated unless the differential is removed explicitly through noncontingent reinforcement in alternating periods of CS and non-CS. Repeated feedings in the absence of the CS presented at the same rate as feedings that had been presented during the CS did not eliminate responding, but responding was eliminated by nondifferential feeding across alternating periods of CS and non-CS. Taken together, these findings suggest a critical role for differential reinforcement, and explicit nondifferential reinforcement, in maintaining and in eliminating conditioned responding, respectively.

Rejection of contiguity does not imply acceptance of contingency. Contingency is a statistical concept that involves the assessment of conditional probabilities over many trials. For that reason, it cannot provide a trial-by-trial account of an acquisition process which, under favorable conditions, can occur in a very small number of trials (see, for example, Jenkins et al., 1981). Nevertheless, the operation of reducing or removing a correlation between CS and
US has major effects, and it is necessary to ask what, if not the contingency itself, might mediate those effects. Contingency imposes a differential or contrast between reinforcing conditions during the CS and the surrounding non-CS periods. This more or less local contrast could be the fundamental condition for CS-US association, and its absence might be the fundamental condition for the elimination of conditioned responding. The dimensions of an effective differential are yet to be identified, but the results we have reviewed suggest that a promising candidate is reinforcer waiting times in the CS as compared with the context. In any case, attention to the events in the CS in relation to neighboring nonCS periods might lead to an alternative to CS-US contiguity and to CS-US contingency, neither of which seems capable of providing a satisfactory account of the associative process in classical conditioning.

\section{REFERENCES}

Ayres, J. B., \& Benedict, J. O. US-alone presentations as an extinction procedure. Animal Learning \& Behavior, 1973, 1, 5-8.

Brookshire, K. H., \& Brackbill, R. M. Formation and retention of conditioned taste aversions and UCS habituation. Bulletin of the Psychonomic Society, 1976, 7, 125-128.

GamzU, E., \& Williams, D. R. Classical conditioning of a complex skeletal response. Science, 1971, 171, 923-925.

Gamzu, E., \& Williams, D. R. Associative factors underlying the pigeon's keypecking in auto-shaping procedures. Journal of the Experimental Analysis of Behavior, 1973, 19, 225-232.

Gibbon, J., \& Balsam, P. D. The spread of association in time. In C. M. Locurto, H. S. Terrace, \& J. G. Gibbon (Eds.), Autoshaping and conditioning theory. New York: Academic Press, 1981.

Hays, W. L. Statistics. New York: Holt, Rinehart, \& Winston, 1981.

Holman, E. W. The effect of drug habituation before and after taste aversion learning in rats. Animal Learning \& Behavior, 1976, 4, 329-332.

Jenkins, H. M., Barnes, R. A., \& Barrera, F. J. Why autoshaping depends on trial spacing. In C. M. Locurto, H. S. Terrace, \& J. G. Gibbon (Eds.), Autoshaping and conditioning theory. New York: Academic Press, 1981.

Lindblom, L. L., \& Jenkins, H. M. Responses eliminated by noncontingent or negatively contingent reinforcement recover in extinction. Journal of Experimental Psychology: Animal Behavior Processes, 1981, 1, 175-190.

Rescorla, R. A., \& Wagner, A. R. A theory of Pavlovian conditioning: Variations in the effectiveness of reinforcement and nonreinforcement. In A. H. Black \& W. F. Prokasy (Eds.), Classical conditioning II: Current research and theory. New York: Appleton-Century-Crofts, 1972.

RILEY, A. L., JAcoBs, W. J., \& LoLondo, V. M. Drug exposure and the acquisition and retention of a conditioned taste aversion. Journal of Comparative and Physiological Psychology, $1976,90,799-807$.

(Manuscript received January 24, 1983; revision accepted for publication May 19, 1983.) 